\title{
TECHNIQUE AND OBSERVATION OF ANGULAR GAIT PATTERNS IN RUNNING
}

K. SYKES, Dip.P.E., D.A.S.P.E., M.Sc.

Senior Lecturer in Physical Education, Chester College, Cheyney Rd., CHESTER

\begin{abstract}
A technique for the biomechanical analysis of running is described with specific reference to the angular displacement patterns of the lower limb. From high speed cine film recording profile views of the running gait, the Thigh, Knee and Ankle angles are measured during one complete cycle. Results are presented in the form of vector-space diagrams, namely Thigh-Knee angle and Knee-Ankle angle cyclograms. The diagrams are interpreted and some experimental observations are presented and discussed.
\end{abstract}

The technique provides a useful research tool and a very good 'teaching asset' for biomechanical studies of movement.

\section{Introduction}

Bipedalism in man has occupied the attention of researchers from early times, though it is with some shame that we note the very recent interests of physical educationists in the field. Walking, at one end of the locomotion continuum, has been thoroughly investigated, however analysis of the running stride has been largely neglected and today it provides subject matter for intricate research.

Dyson (1970) views this 'classical athletic sport' as both simple and difficult; simple from the point of view that it is an instinctive and natural skill to all but the unfortunate; and difficult because of the complexity of its mechanical structure. Styles are as numerous as the individuals who run, for every runner brings to his style the modifying factors of anatomic structure, body proportions, strength and flexibility, posture and his own interpretation of some fundamental phase of the skill.

\section{Review of Literature}

Since the earliest work carried out by the Weber brothers in 1836, many methods of recording the mechanical analysis of human movement have been devised which are of great interest to researchers, orthopaedic surgeons, physiotherapists and physical educationists. Cine photography is a means of recording motion, and individual frames can be enlarged, the displacement of the limbs measured from which various computations may then be made.

The glass walkway (Eberhart and Inman, 1951) was designed for the study of three-dimensional aspects, the vertical plane being filmed upward through the walkway.
Stroboscopy (Jardine, 1965) has the advantage of giving a series of images on the one photograph.

High speed triaxial cinematography (Jokl, 1968) is now widely used in movement analysis, using overhead views to give the third dimension.

Various electronic techniques of recording movement have been developed.

The temporal factors walkway (Contini, 1964) enables the phasic periods of locomotion to be recorded.

Electrogoniography (Jokl, 1968) is a technique for recording the angular displacements at the various joints.

Tachography, accelerometry and the force platform (Jokl, 1968) are means of measuring the velocities, accelerations and forces generated by the body.

Electromyography (Basmajian, 1967) enables muscular activity to be studied by recording the changes in electrical potential of muscle fibres which accompany contractions.

Movement Notation is a method of recording and representing movements by means of symbols. Because the notation is based on a scientific analysis of the elements of movement, all forms, ranging from the simplest to the most complex may be written. However, the design of a notation system for the use in physical education, science and technology must be such as to facilitate, not only the recording of all forms of movement, but also to make possible creative work, research and analysis. (Hutchinson, 1955; Causley, 1967; Laban, 1956; Hopper, 1969). 


\section{Angular Gait Patterns}

The Angle-Angle graphical representation of the walking gait was devised by Grieve (1968), who was looking for a method of movement notation which could be used in pathological cases to record improvements in the walking gait of patients being treated for various conditions (arthritis, rheumatism, reparation from fractures, muscular dystrophy, etc.). He wanted a precise notation which would be capable of duplication and comparison. By measuring limb angles from film and plotting them against time, one can follow the course of a particular joint movement. In addition, by plotting one angle against an adjacent joint angle, one can represent instantaneous postures during the walking cycle. This method of producing a cyclic graph has the advantage of overlay, and as a result, quick checks on patient progress may be made. Once the physician, orthopaedic surgeon and physiotherapist become 'au fait' with interpretation of these angle-angle diagrams, then appropriate treatment may be quickly and readily prescribed.

The technique has obvious transfer into any movement activity and becomes particularly useful in analysing cyclic activities. Its use is certainly not restricted to medicine, but may be employed most fortuitously into the realm of the physical educationist.

\section{Technique and Analysis:}

Angle-angle diagrams of the running gait require the following measurements from high speed cine film recording profile views -

1. Thigh angle - the angle between the line of the thigh and the vertical.

2. Knee angle - the angle between the extended line of the thigh and the line of the lower leg.

3. Ankle angle - the angle between the line of the lower leg and the sole of the foot. (Fig. 1).

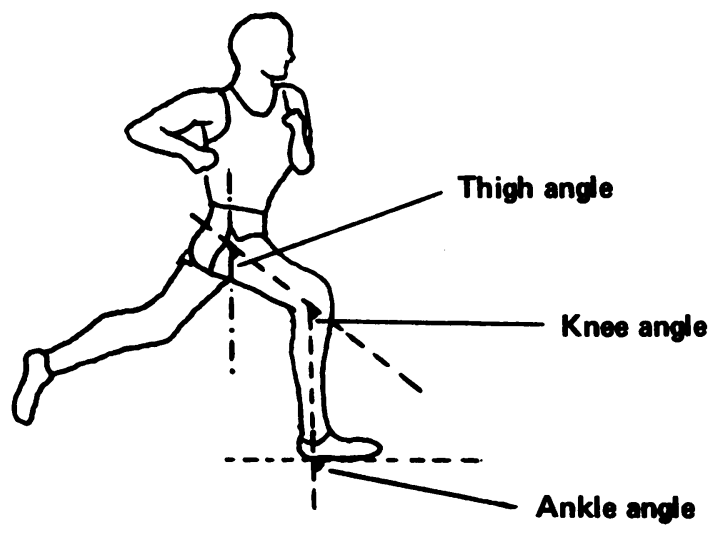

Fig. 1. Graphs of Thigh-Knee angle and Knee-Ankle angle may then be plotted and observed for analysis.
Knøeo

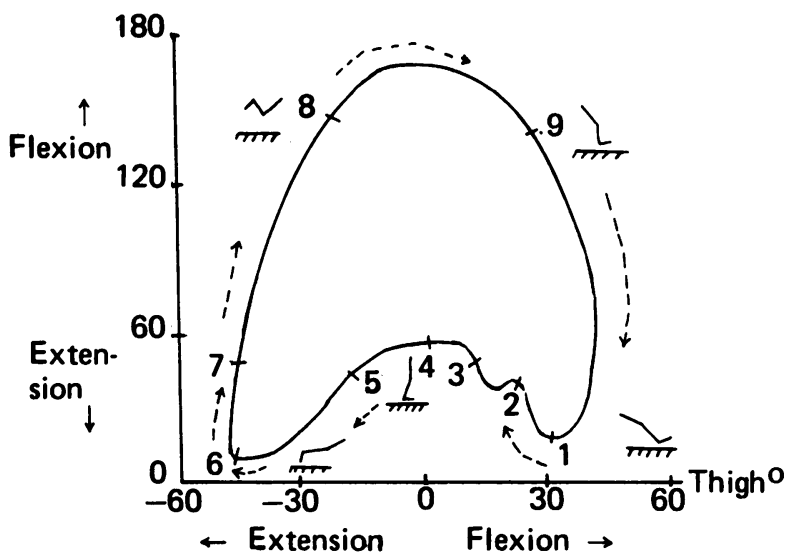

Fig. 2. Thigh-Knee angle diagram Fig. 2:

Analysis:

1. Completion of the forward swing

1-2 Knee commences flexion, reducing the forward velocity of the foot relative to the ground in preparation for footstrike

2. Footstrike (FS)

2-3 Brief phase immediately after footstrike when knee tends to lock - possibly as a result of patellar tendon reflex action initiated by shock of impact, thereby causing momentary uncontrollable quadriceps tension (Brookes et al. 1971)

3.4 Controlled increase in knee angle as the downward momentum of the body is absorbed and the $C$ of $G$ coasts forward until above the supporting foot. The thigh angle remains virtually constant.

4.5 Knee angle remains constant whilst the thigh decreases to the vertical as the body rotates over the foot

5- Heel lift

5-6 Thrust phase - thigh becomes negative and the knee extends rapidly

6- Toe off (TO)

6-7 Knee flexion commences for recovery stage

7-8 Thigh begins its forward swing whilst the knee flexes rapidly, thereby reducing the moment of inertia about the hip

8-9 Thigh flexes as the knee remains flexed, thus completing the recovery with minimum moment of inertia

9-1 Thigh becomes stabilized as the knee extends to straighten the leg in its forward swing 


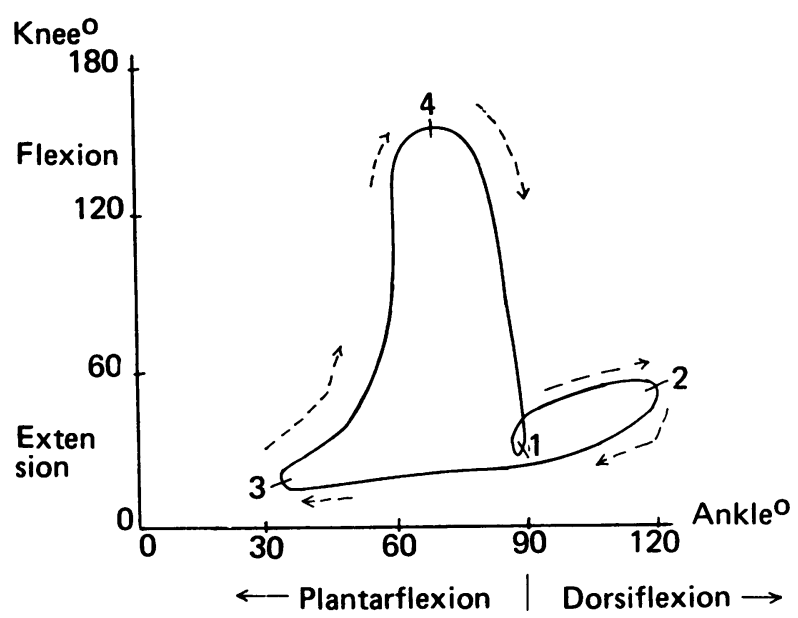

Fig. 3. Knee-Ankle angle diagram

Analysis:

1. Footstrike

1-2 Dorsiflexion of the foot as lower leg pivots forward over the ankle

2. Heel lift

2-3 Vigorous plantarflexion of the foot during the thrust phase

3. Toe off (TO)

3-4 Rapid knee flexion during the recovery stage, the foot remains slightly plantarflexed

4-1 The lower leg swings forward (knee extension), the foot returns to 900 which is approximately retained for footstrike

The two analyses give approximate interpretations of typical cyclograms. However experimental work serves to indicate the variations on this theme due to speed, gradient, individual and environmental factors from which implications, discussion and conclusions may be drawn.

\section{Experimental observations:}

1. Subject - P. SNELL, New Zealand, Triple Olympic Gold medallist and World Record Holder at $880 \mathrm{yd}$ and Mile. Filmed in 1972 at the Human Performance Laboratory, University of Technology, Loughborough.
Speed (Kph) 23.232 .2

Cycle Time (sec) 0.680 .50

Stride length (metrs) 2.332 .35

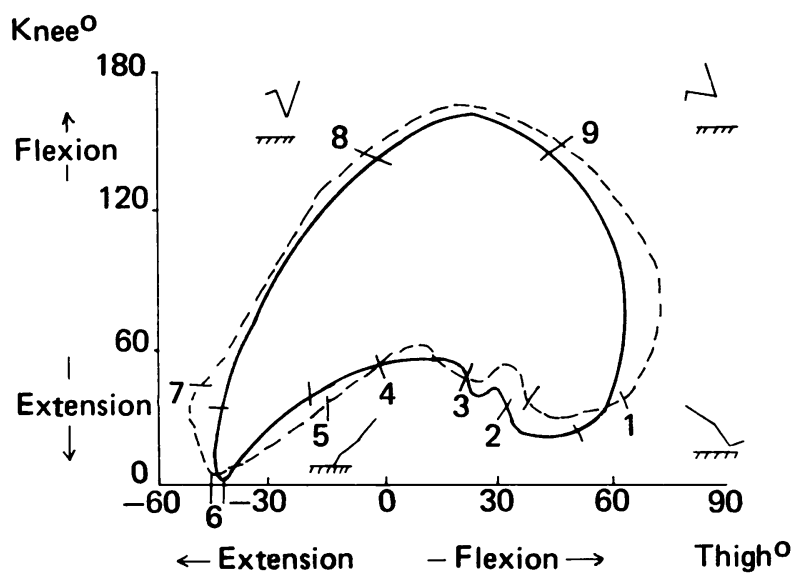

Fig. 4. Thigh-Knee angle diagram to compare angular gait patterns at two different running speeds.

Analysis:

a. Thigh has larger range of movement at the faster speed, Knee range is approx. the same at both speeds.

b. At completion of Forward Swing (1) Thigh flexion is $20^{\circ}$ greater and Knee extension $15^{\circ}$ less at the faster speed.

c. At Footstrike (2) Thigh flexion is slightly greater at faster speed.

d. Support phase (3-6) quite similar in angular characteristics.

e. Knee is almost completely extended at the end of the thrust phase (6) at both speeds - the hallmark of good technique!

f. Extension of Thigh and Knee at Toe Off (6) are approx. the same for both speeds - an unusual feature suggested as a training effect.

g. Continuation of Thigh extension accompanied by rapid Knee flexion after Toe Off at the faster speed a powerful thrust action.

h. Recovery patterns very similar (7-9).

i. Greater Thigh flexion during Forward Swing (9-1) at faster speed.

j. An unusual feature of the two runs was that despite a difference of $9.0 \mathrm{kph}$ in speed and 0.18 sec difference in Cycle Time, there was NO significant change in Stride Length.

By similar overlay of the Knee-Ankle angle cyclograms one may observe that a greater range of ankle movement is present in faster running - plantarflexion is increased by some $10^{\circ}$ and dorsiflexion during the support phase by a similar amount. 
2. Subject: JKR - physical education student of average running ability

Speed (Kph) 22.330 .4

Cycle time (secs) 0.640 .50

Stride length (mtrs) 1.842 .01

Knee ${ }^{\circ}$

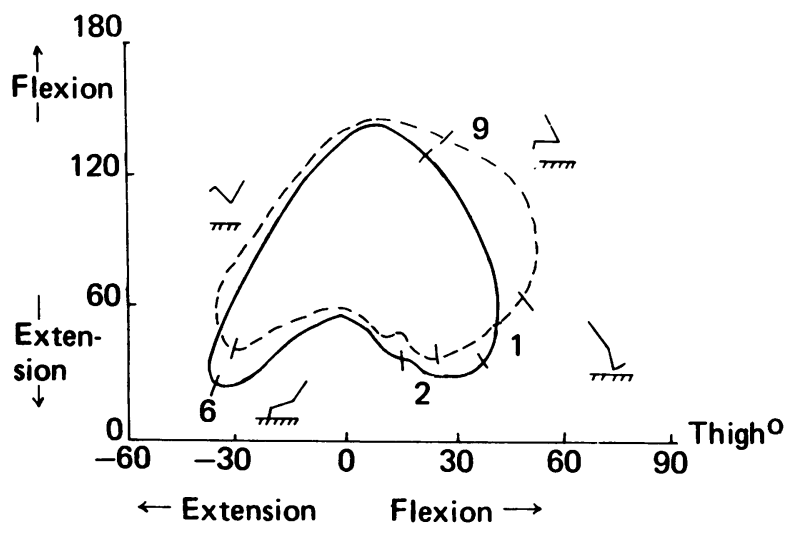

Fig. 5. Thigh-Knee angle diagram to compare angular gait patterns at two different running speeds.

\section{Analysis:}

a. Thigh has larger range of movement at faster speed, Knee has larger range at slower speed.

b. At completion of forward swing (1) Thigh flexion is $12^{\circ}$ greater and Knee extension $25^{\circ}$ less at the faster speed.

c. At Footstrike (2) Thigh flexion is $10^{\circ}$ greater at the faster speed.

d. Knee extension at Toe Off (6) - end of thrust phase - is $20^{\circ}$ less at faster speed, perhaps due to a time factor.

e. During forward swing (9-1) Thigh flexion is greater at faster speed, Knee extension is far more rapid at slower speed.

f. Footstrike (2) at slower speed occurs with only $15^{\circ}$ of Thigh flexion!

g. By way of comparison with $P$. Snell the total range of angular movement displayed by JKR is far less for comparable speeds; Knee extension is relatively limited during thrust, Thigh flexion during forward swing is also limiting; stride length is consequently shorter and more variable.

The Knee-Ankle diagram of JKR shows poor Ankle flexibility and hence a reduced effective thrust.
3. Subject: P. Snell, involved in an experiment to investigate the biomechanical differences which may exist between road and treadmill running. (Sykes, 1972).

Road T/Mill

Speed (Kph) 25.025 .0

Cycle time (secs) 0.660 .63

Stride length (mtrs) 2.332 .18

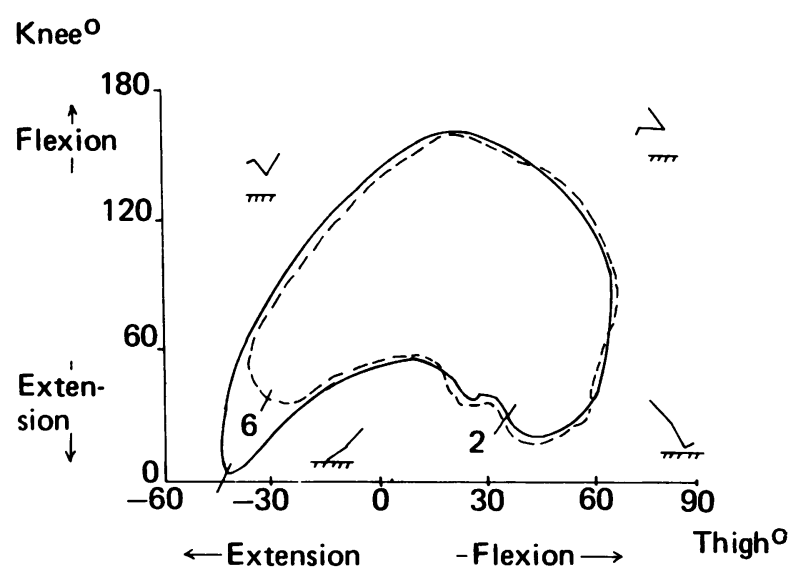

Fig. 6. Thigh-Knee angle diagram to compare angular gait patterns of road and treadmill running at the same speed.

\section{Analysis :}

a. Apart from the thrust phase (6) the two cyclograms are similar in angular displacement patterns.

b. During the thrust phase the Knee becomes almost completely extended on the road run, whilst $35^{\circ}$ of flexion are observed on the treadmill run.

c. Hip extension during thrust is also less on the treadmill run.

d. Stride length is shorter, Cycle time is faster on the treadmill run. 
Road T/Mill

Speed (Kph) 25.025 .0

Cycle time (secs) 0.660 .63

Stride length (mtrs) 2.332 .18

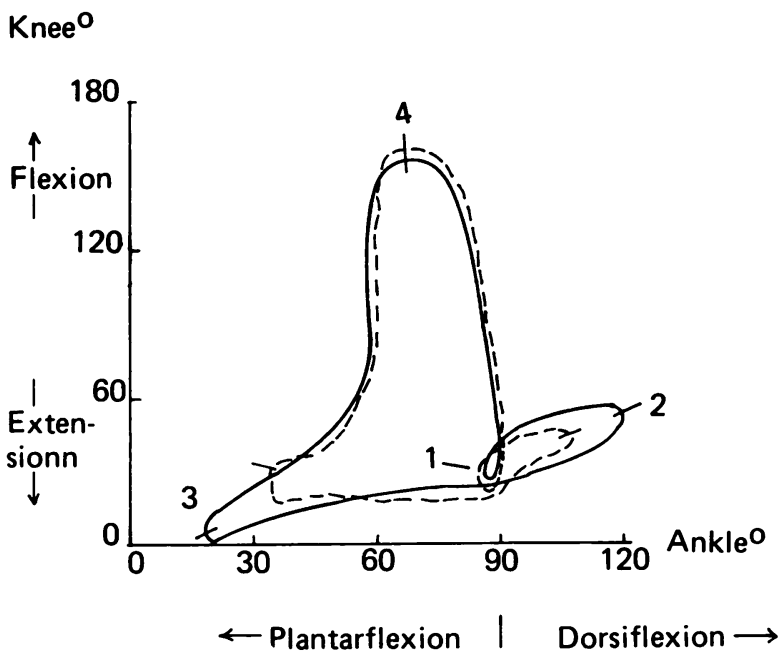

Fig. 7. Subject: P. Snell. Knee-Ankle angle diagram to compare angular gait patterns of road and treadmill running at the same speed.

Analysis:

a. Ankle displays greater range of movement in road running.

b. During support phase, ankle has $15^{\circ}$ more dorsiflexion at Heel lift (2) in road running. c. During thrust phase, ankle shows greater plantarflexion; $20^{\circ}$ greater at Toe Off (3) in road running.

d. As a powerful thrust is largely dependent upon Knee and Ankle extension, one may observe the limited range during this phase in treadmill running.

\section{Discussion and Conclusions}

Extensive use has been made of angle-angle diagrams in these studies in preference to the conventional angletime graphs. Running is a cyclic activity and the continuity is emphasized by plotting the result as a cycle. Angle-angle diagrams emphasize the relationships between angles more clearly than the separate plots and with practice convey an impression of changing inertias, and contributions to horizontal and vertical movements. A time scale may be added to the cycle thus giving information about the timing of phases; more detailed work may be observed with the alliance of anthropometric data, physiological and electromyographical studies.

With the ease of application and the absence of expensive goniometers the method provides a very good 'teaching asset', giving a step-by-continued-step study of a movement.

Running form is the result of both inherited and developmental factors. As physical educationists we have no control over the former, but correct structuring of our athletic programmes on the mechanical principles and observations outlined would result in a more accurate channelling of talents and effort toward a greater measure of success by those who are fortunate enough to possess the necessary, inherited characteristics.

\section{REFERENCES}

Basmajian, J. V. Muscles Alive. Williams \& Wilkins Co., Baltimore, 1967.

Brookes, C., Pantlin, C., Wilson, K. The Science of Human Movement. Part 2. Unpublished Thesis, C.A.S. in P.E. University of Leeds, 1971.

Causley, M. An Introduction to Benesh Movement Notation. Max Parish: London, 1967.

Contini, R., Gage, H., Drillis, R. Human Gait Characteristics: Biomechanics and related Bioengineering Topics. Ed. by Kenedi, R. M., Pergamon, 1964.

Dyson, G. H. G. The Mechanics of Athletics. London Uni. Press, 1970.

Eberhart, H. D. and Inman, V. T. An Evaluation of Experimental Procedures used in a Fundamental study of Human Locomotion. Ann.New York Acad.Sci., 51, 1213-1228, 1951.

Grieve, D. W. Gait Patterns and the Speed of Walking. Bio-medical Engineering, March, 1968.

Hopper, B. J. Characteristics of the Running Stride. Roy. Can. Leg.Cchg.Rev., Sept., 1969. 
186

Jardine, J. T. Photography and Physics. Visual Education, March, 1965.

Jokl, E. (Ed.) Biomechanics: Technique of Drawings of Movement and Movement Analysis. S. Karger, Basel, 1968.

Laban, R. Principles of Dance and Movement Notation. Macdonald \& Evans, London, 1956.

Slocum, D. B. and James, S. L. Biomechanics of Running. J.A.M.A. 205, 721-728, 1968.

Sykes, K. An Investigation into the Biomechanical Differences which may exist between Road and Treadmill Running. Unpublished dissertation, University of Technology, Loughborough, 1972. 